\title{
Angular Dependences of ESR Parameters in Antiferroquadrupolar Phase of $\mathrm{CeB}_{6}$
}

A.V. Semeno ${ }^{a, *}$, M.I. Gilmanov ${ }^{a, b}$, N.E. Sluchanko ${ }^{a}$, V.N. Krasnorussky ${ }^{a}$, N.Y. Shitsevalova ${ }^{c}$, V.B. Filipov ${ }^{c}$, K. Flachbart ${ }^{d}$ AND S.V. DemisheV ${ }^{a}$

${ }^{a}$ Prokhorov General Physics Institute, RAS, 119991, Vavilov Str. 38, Moscow, Russia

${ }^{b}$ Moscow Institute of Physics and Technology, 141700, Institutsky lane 9, Dolgoprudniy, Russia

${ }^{c}$ Frantsevich Institute for Problems of Materials Science of NASU, Krzhyzhanovskii Str. 3, 03680 Kiev, Ukraine

${ }^{d}$ Institute of Experimental Physics, SAS, Watsonova Str. 47, SK-04001 Košice, Slovak Republic

Angular dependences of ESR line parameters ( $g$-factor and linewidth $\Delta H$ ) were experimentally explored in the antiferroquadrupolar phase of heavy fermion system $\mathrm{CeB}_{6}$ at $T=1.8 \mathrm{~K}$. The data were obtained in two experimental geometries with different mutual directions of the wavevector $\boldsymbol{k}$ and the external magnetic field $\boldsymbol{H}$ at frequency of $f=60 \mathrm{GHz}$. A $g$-factor anisotropy was found: while $g$-factors for [110] and [111] directions are close to each other $(g \approx 1.6)$, it is considerably higher $(g \approx 1.75)$ for [100]. The obtained angular dependence $g(\Theta)$ was compared with the theoretically predicted $g$-factor behavior for the $\Gamma_{8}$ state of $\mathrm{Ce}^{3+}$ ion in antiferroquadrupolar phase of $\mathrm{CeB}_{6}$. It turns out that the experimental $g$-factor is considerably smaller at all angles than the theoretically calculated limits $(2<g<2.2)$ and, moreover, it has a different symmetry. This result together with the strong linewidth anisotropy $\Delta H(\Theta)$ demonstrates that for the relevant description of experimental data the theoretical model should take into account also the interaction of $\mathrm{Ce}^{3+}$ ion with itinerant $5 d$ electrons.

DOI: $10.12693 /$ APhysPolA.131.1060

PACS/topics: $76.30 .-\mathrm{v}, 76.30 . \mathrm{Kg}$

\section{Introduction}

Electron spin resonance (ESR) in heavy fermion compound $\mathrm{CeB}_{6}[1-3]$ is a puzzling phenomenon forming new aspects in the investigation of the exotic low temperature state of this system. Strong exchange interaction of local moments with conduction electrons prevents the observation of the resonance behavior of $\mathrm{Ce}^{3+}$ ions in metals and only few systems exhibiting ESR have been reported so far ([4] and references therein). The peculiar place of $\mathrm{CeB}_{6}$ in this series is related with the ESR behavior at the onset of antiferroquadrupolar (AFQ) order in it. The AFQ phase at zero magnetic field develops below $T_{Q}=3.2 \mathrm{~K}$. Further temperature decrease transfers this compound into AFM phase below $T_{N}=2.4 \mathrm{~K}$. Magnetic field application stabilizes the AFQ phase causing an increase of $T_{Q}$ as well as a suppression of AFM order below $T_{N}$. The experimental observation of ESR in $\mathrm{CeB}_{6}$ [1-3] induced a novel theoretical approach describing ESR in the AFQ phase $[5,6]$. It is based on the consideration of $\Gamma_{8}$ state of $\mathrm{Ce}^{3+}$ ions forming two orbitally ordered sublattices. This model explains the observation of a single ESR line instead of four lines expected for $\Gamma_{8}$ state and it predicts an angular dependence of the $g$-factor. It is worth noting that up to now ESR in $\mathrm{CeB}_{6}$ has been studied exclusively for magnetic field applied in [110] crystallographic direction [1-3] and that measurements of ESR anisotropy could not just examine the relevance of the theory but also clarify the magnetic state of $\mathrm{Ce}^{3+}$ ion in $\mathrm{CeB}_{6}$.

*corresponding author; e-mail: semeno@lt.gpi.ru

\section{Experimental details}

Single crystals of $\mathrm{CeB}_{6}$ were grown by the floatingzone method. Details concerning samples preparation technique can be found elsewhere [7].

For high frequency $(f=60 \mathrm{GHz})$ cavity measurements three cylindrical plates were cut with surfaces perpendicular to high symmetry directions [100], [111] and [110]. Magnetic resonance experiments were carried out with the use of homemade spectrometer based on Agilent E8361C Network Analyzer at temperature $T=1.8 \mathrm{~K}$. The method based on the comparative analysis of resonator magnetoabsorption and magnetoresistive $\rho(H)$ dependences was applied to obtain ESR curves in units of magnetic permeability [8].

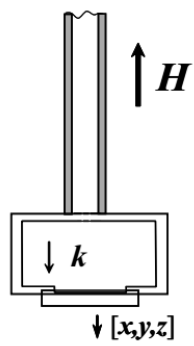

a) $k \| \boldsymbol{H}$

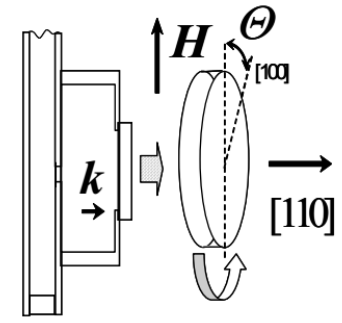

b) $\boldsymbol{k} \perp \boldsymbol{H}$
Fig. 1. Experimental geometries of ESR experiments with wavevectors $\boldsymbol{k}$ parallel (a) and perpendicular (b) to external magnetic field $\boldsymbol{H}$. 


\section{Results and discussion}

Two different experimental schemes may be applied to ESR measurements of metallic samples in solenoids. In the first experimental geometry (Fig. 1) used for $\mathrm{CeB}_{6}$ in this work, the sample surface is perpendicular to the external magnetic field $H$ (Fig. 1a). This standard scheme allows to obtain ESR spectra for samples cut in [100], [111] and [110] directions, and it can be used also for temperature measurements. For the purpose of detailed angular measurements of ESR parameters in metallic samples a novel experimental setup was developed (Fig. 1b). In this case the magnetic field is parallel to the sample surface and there is an ESR-active surface area for every field direction during sample rotation. Due to the fact that only a part of the entire sample surface participates in the resonance, the ESR signal is in this case lower and reliable results on $\mathrm{CeB}_{6}$ are available only at the lowest temperatures. In this experiment the axis of the flat cylindrical sample is parallel to [110] direction (Fig. 1b). Thus, discrete sample rotation around [110] direction (Fig. 1b) allows to measure ESR spectra for different angles $\Theta$ including all principal crystallographic directions [110] $\left(\Theta=90^{\circ}\right)$, [100] $\left(\Theta=0^{\circ}\right)$ and [111] $\left(\Theta \approx 56^{\circ}\right)$. It is necessary to notice that the above experimental schemes correspond to different geometries of the microwave wavevector $\boldsymbol{k}$ relatively to magnetic field $\boldsymbol{H}: \quad \boldsymbol{k} \| \boldsymbol{H}$ in the first case and $\boldsymbol{k} \perp \boldsymbol{H}$ in the second (Fig. 1). The results then differ in resonance conditions: while in $\boldsymbol{k} \| \boldsymbol{H}$ case the microwave absorption is defined by two circularly polarized waves $\mu^{+}=\mu+\mu_{\alpha}$ and $\mu^{-}=\mu-\mu_{\alpha}$, the effective magnetic permeability in the case of $\boldsymbol{k} \perp \boldsymbol{H}$ is $\mu_{\mathrm{eff}}=\mu-\mu_{\alpha}^{2} / \mu$, where $\mu$ and $\mu_{\alpha}$ are elements of the magnetic permeability tensor [9]. In the present study special attention was paid to correct data analysis and to the conformity of results obtained in two above experimental schemes.

The experiment in the standard scheme (Fig. 1a) on three samples with surfaces perpendicular to [110], [111] and [100] has demonstrated an anisotropy of $g$-factor $g \approx 1.6$ for [110], [111] and $g \approx 1.75$ for [100]. The corresponding values of resonance fields $\left(H_{\text {res }} \approx 26.6 \mathrm{kOe}\right.$ for [110], [111] and $H_{\text {res }} \approx 24.3 \mathrm{kOe}$ for [100]) lie higher than AFM-AFQ transition fields $\left(H_{0} \approx 21 \mathrm{kOe}\right.$ for [100] [10, 11] and $H_{0} \approx 11 \mathrm{kOe}$ for [110] and [111] [12]) and therefore ESR is entirely settled in the AFQ phase diagram area. In this case the theoretical model $[5,6]$ should be applicable to the experimental data and it is reasonable to make their comparative analysis. The angular dependence obtained in the rotating resonator (Fig. 1b) is shown in Fig. 2. It is visible that the direction [100] corresponds to the maximum of $g(\Theta)$ dependence, then $g(\Theta)$ quickly drops apart from this direction. The theoretical model $[5,6]$ contains an internal parameter $\phi$ which is the angle between two quadrupolar sublattices which provides different $g(\Theta)$ for every $\phi$. However, according to $[5,6]$ it is possible to localize the area of $g(\Theta)$. It is restricted by limit dependences $g(\Theta)$ (for $\phi=0$ ) and $g(\Theta)$ (for $\phi=\pi / 2$ ) shown in

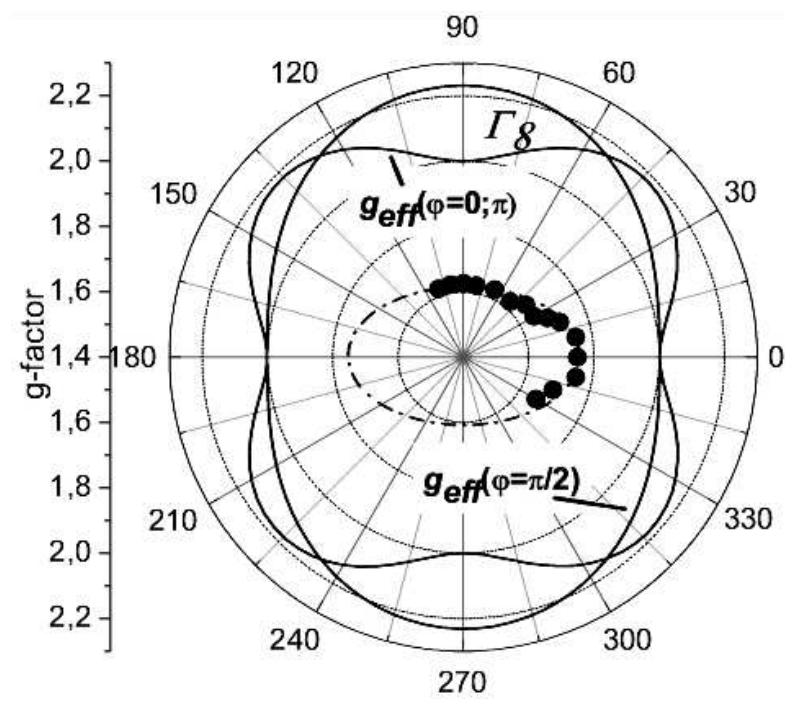

Fig. 2. Angular dependence of ESR $g$-factor $g(\Theta)$ in $\mathrm{CeB}_{6}$ at $T=1.8 \mathrm{~K}$ (circles). Lines show calculated $g(\Theta)$ dependences for the $\Gamma_{8}$ state of $\mathrm{Ce}^{3+}$ ion in AFQ phase at different angles $\phi$ between sublattices.

Fig. 2. All other $g(\Theta)$ curves are located in the area between these limit cases. The experiment unambiguously demonstrates that the theoretical model which takes into account exclusively crystal field effects in the AFQ state fails to describe the experimental data. First, experimental $g$-factors $(1.6<g<1.75)$ are considerably lower than the theoretically calculated ones $(2<g<2.2)$ for all other angle parameters $\Theta$ and $\phi$. Moreover, the $g(\Theta)$ dependences have qualitatively different symmetry than the theoretically predicted.

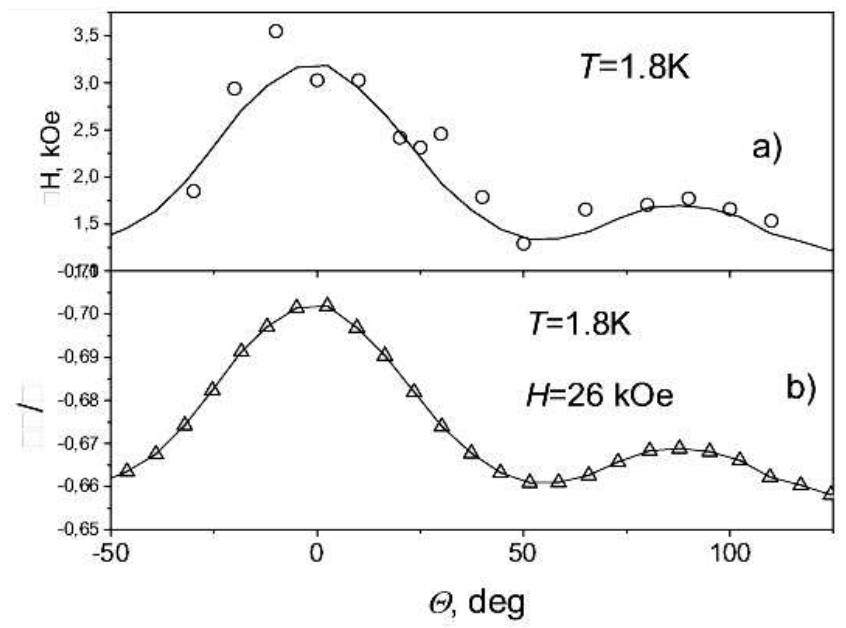

Fig. 3. Angular dependence of (a) ESR linewidth $\Delta H(\Theta)$, (b) magnetoresistance at the resonance field in $\mathrm{CeB}_{6}$ at $T=1.8 \mathrm{~K}$. Lines are guides for the eye.

In order to reveal additional physical mechanisms which are able to affect the ESR behavior one can analyze 
the ESR linewidth angular dependence $\Delta H(\Theta)$ (Fig. 3). It is well correlated with $g(\Theta)$ exhibiting a maximum value at $\Delta H \approx 3 \mathrm{kOe}$ in [100] which is much higher than the average value $\Delta H \approx 1.6 \mathrm{kOe}$ in other directions. This fact may indicate a strong spin relaxation in this direction caused by the interaction with itinerant $d$ electrons. To check this assumption one can compare the linewidth behavior with transport angular properties. Indeed, the analysis shows good correlation between $\Delta H(\Theta)$ and the magnetoresistivity angular dependence $\Delta \rho / \rho(\Theta)$ measured at the resonance field $H \approx 26 \mathrm{kOe}$ (Fig. 3).

This result confirms the above statement of the role of $4 f-5 d$ interaction in ESR behavior which results, first, into a strong relaxation of $\mathrm{Ce}^{3+}$ magnetic moments and, second, into an additional contribution to the itinerant electrons scattering taking place in [100] direction. It is also important to emphasize the considerable anisotropy of spin relaxation which restricts the application of standard isotropic models usually applied for interactions like the Korringa mechanism [13]. Thus, the current study shows the necessity of novel theoretical approaches to this problem.

\section{Conclusions}

A comparative analysis of the angular dependence of ESR $g$-factor $g(\Theta)$ with theoretical calculations made for the $\Gamma_{8}$ state of $\mathrm{Ce}^{3+}$ ion in $\mathrm{AFQ}$ phase of $\mathrm{CeB}_{6}$ has demonstrated the failure of predictions both in the $g$ factor magnitude and its symmetry. The correlation between ESR linewidth and magnetoresistivity angular dependences allows to suggest the important role of $4 f$ $5 d$ interactions in the formation of the low temperature magnetic state of $\mathrm{Ce}^{3+}$ ions in $\mathrm{CeB}_{6}$.

\section{Acknowledgments}

This work was supported by the project RFBR 14-0200800 as well as by programs of the Russian Academy of Sciences "Electron spin resonance, spin-dependent electronic effects and spin technologies" and "Electron correlations in strongly interacting systems". The support of VEGA $2 / 0032 / 16$ is acknowledged, too.

\section{References}

[1] S.V. Demishev, A.V. Semeno, A.V. Bogach, N.A. Samarin, T.V. Ishchenko, V.B. Filippov, N.Yu. Shitsevalova, N.E. Sluchanko, Phys. Status Solidi B 242, R27 (2005).

[2] S.V. Demishev, A.V. Semeno, A.V. Bogach, N.A. Samarin, T.V. Ishchenko, V.B. Filipov, N.Yu. Shitsevalova, N.E. Sluchanko, Phys. Rev. B 80, 245106 (2009).

[3] S.V. Demishev, A.V. Semeno, A.V. Bogach, Yu.B. Paderno, N.Yu. Shitsevalova, N.E. Sluchanko, J. Magn. Magn. Mater. 300, E534 (2006).

[4] C. Krellner, T. Forster, H. Jeevan, C. Geibel, J. Sichelschmidt, Phys. Rev. Lett. 100, 066401 (2008).

[5] P. Schlottmann, Phys. Rev. B 86, 075135 (2012).

[6] P. Schlottmann, J. Appl. Phys. 113, 17E109 (2012).

[7] N.Yu. Shitsevalova, Yu. Paderno, AIP Conf. Proc. 231, 326 (1991).

[8] A.V. Semeno, V.V. Glushkov, A.V. Bogach, N.E. Sluchanko, A.V. Dukhnenko, V.B. Fillippov, N.Yu. Shitsevalova, S.V. Demishev, Phys. Rev. B 79, 014423 (2009).

[9] J.A. Young, E.A. Uehling, Phys. Rev. 94, 544 (1954).

[10] T. Yamamizu, M. Endo, M. Nakayama, N. Kimura, H. Aoki, S. Kunii, Phys. Rev. B 69, 014423 (2004).

[11] S. Nakamura, T. Goto, S. Kunii, J. Phys. Soc. Jpn. 64, 3941 (1995).

[12] N.E. Sluchanko, A.V. Bogach, V.V. Glushkov, S.V. Demishev, V.Yu. Ivanov, M.I. Ignatov, A.V. Kuznetsov, N.A. Samarin, V. Semeno, N.Yu. Shitsevalova, J. Exp. Theor. Phys. 104, 120 (2007).

[13] S.E. Barnes, Adv. Phys. 30, 801 (1981). 